\title{
Manual MRI parcellation of the frontal lobe
}

\author{
Marin E. Richardson ${ }^{\mathrm{a}}$, Deana Crocetti ${ }^{\mathrm{a}}$, Jacqueline A. Clauss ${ }^{\mathrm{a}}$, Michael A. Kraut ${ }^{\mathrm{f}}$, Stewart H. \\ Mostofskya,b,d, and Walter E. Kaufmanna,b,c,d,e,f, ${ }^{*}$ \\ a Kennedy Krieger Institute, Baltimore, MD, USA \\ b Department of Neurology, Johns Hopkins University School of Medicine, Baltimore, MD, USA \\ c Department of Pathology, Johns Hopkins University School of Medicine, Baltimore, MD, USA \\ d Department of Pediatrics, Johns Hopkins University School of Medicine, Baltimore, MD, USA \\ e Department of Psychiatry and Behavioral Sciences, Johns Hopkins University School of Medicine, \\ Baltimore, MD, USA \\ f Department of Radiology and Radiological Science, Johns Hopkins University School of Medicine, \\ Baltimore, MD, USA
}

\begin{abstract}
The ability to examine associations between neuropsychiatric conditions and functionally relevant frontal lobe sub-regions is a fundamental goal in neuropsychiatry, but methods for identifying frontal sub-regions in MR (magnetic resonance) images are not well established. Prior published techniques have principally defined gyral regions that do not necessarily correspond to known functional divisions. We present a method in which sulcal-gyral landmarks are used to manually delimit functionally relevant regions within the frontal lobe: primary motor cortex, anterior cingulate, deep white matter, premotor cortex regions (supplementary motor complex (SMC), frontal eye field and lateral premotor cortex) and prefrontal cortex (PFC) regions (medial PFC, dorsolateral PFC (DLPFC), inferior PFC, lateral orbitofrontal cortex (OFC) and medial OFC). Feasibility was tested by applying the protocol to brain MR data from 15 boys with ADHD and 15 typically developing controls, 8-12 years old. Intra- and inter-rater intraclass correlation coefficients were calculated using parcellation volumes from a subset of that group. Inter-rater results for the 22 hemisphere specific sub-regions ranged from 0.724 to 0.997, with all but six values above 0.9. Boys with ADHD showed significantly smaller left hemisphere SMC and DLPFC volumes after normalization for total cerebral volume. These findings support the method as a reliable and valid technique for parcellating the frontal lobe into functionally relevant subregions.
\end{abstract}

\section{Keywords}

ADHD; Children; Cortex; Prefrontal; Premotor; Segmentation; Supplementary Motor Complex

Corresponding Author: Walter E. Kaufmann, Kennedy Krieger Institute, 3901 Greenspring Ave., Baltimore, MD 21211, Phone: 443-923-2789, Fax: 443-923-7696, Email: kaufmann@kennedykrieger.org.

Publisher's Disclaimer: This is a PDF file of an unedited manuscript that has been accepted for publication. As a service to our customers we are providing this early version of the manuscript. The manuscript will undergo copyediting, typesetting, and review of the resulting proof before it is published in its final citable form. Please note that during the production process errors may be discovered which could affect the content, and all legal disclaimers that apply to the journal pertain. 


\section{1.) INTRODUCTION}

The frontal lobe is the region of the brain associated with executive control and planning, including functions such as response inhibition and working memory (Tekin \& Cummings, 2002; Alvarez \& Emory, 2006). The frontal lobe is also involved in motivational aspects of behavior and high-order motor control (Tekin et al., 2002; Ashe et al., 2006; Graziano \& Aflalo, 2007). Due to this wide range of complex brain functions, abnormalities in frontal lobe structure and function have been hypothesized to contribute to many neuropsychiatric disorders, including obsessive-compulsive disorder (OCD), schizophrenia, depression and bipolar disorder (Tekin et al., 2002; Yamasue et al., 2004;Suzuki et al., 2005; Shad et al., 2006). Frontal lobe abnormalities have been particularly emphasized in the pathophysiology of developmental disorders such as Down syndrome (Pinter et al., 2001; Porter et al., 2007), Rett Syndrome (Carter et al., 2008b), Fragile X syndrome (Kates et al., 2002a; Gothelf et al., 2008), Williams syndrome (Reiss et al., 2004; Porter et al., 2007), Neurofibromatosis Type 1 (Cutting et al., 2002; Greenwood et al., 2005), idiopathic Autism (Acosta \& Pearl, 2004; Courchesne et al., 2007), Tourette Syndrome (Fredericksen et al., 2002; Marsh et al., 2007) and Attention-Deficit/ Hyperactivity Disorder (ADHD) (Mostofsky et al., 2002; Sowell et al., 2003; Nigg \& Casey, 2005; Shaw et al., 2006; Kelly et al., 2007; Shaw et al., 2007). In view of the size and functional heterogeneity of the frontal lobe (Fuster, 1997), it is likely that abnormalities in distinct functional regions are preferentially associated with a particular disorder or with a specific aspect of that disorder.

In an attempt to develop a tool for functionally relevant morphological and volumetric assessments of the frontal lobe and its sub-regions, we have developed a manual frontal parcellation protocol that relies on prominent and consistent sulcal-gyral landmarks to delineate functionally distinct regions. While gyral-sulcal landmarks define cytoarchitectonically- and functionally-relevant discrete regions in most cortical areas (e.g., angular gyrus/area 39 of Brodmann/posterior inferior parietal lobule) (Zilles et al., 1990; Geyer et al., 2000; Tekin et al., 2002), the major frontal gyri traverse multiple cytoarchitectonic and functional regions (Sanides, 1972). Therefore, frontal gyral regions may not correspond to functional regions and identification of minor frontal gyri/sulci is neccessary for defining functional components. Previous protocols have often used only the most prominent sulcal landmarks due to concerns about rater reliability and image resolution, preserving most of the major frontal gyri as frontal subdivisions and leading therefore to predominantly anatomical parcellation schemes (Wible et al., 1997; Buchanan et al., 1998; Crespo-Facorro et al., 1999,2000; Convit et al., 2001; Howard et al., 2003; Desikan et al., 2006; John et al., 2006). For example, the approach proposed by Rademacher and colleagues in 1992 and its subsequent revisions (e.g. Kennedy, 1998 \#1927) defined 14 frontal lobe sub-regions with boundaries defined by multiple sulci and landmark-based planes extended through the brain volume (cut planes), using techniques similar to ours (Kates et al., 2002b). However, the resulting parcellation units were predominantly gyri or portions of them with limited correspondence to functional regions (Rademacher et al., 1992; Kennedy et al., 1998). Significantly, two of the Rademacher subregions, corresponding to the superior and middle frontal gyri, included several Brodmann areas each, including regions from both prefrontal and premotor areas whose divergent functions are well established (Tekin et al., 2002; Alvarez et al., 2006; Graziano et al., 2007).

In 2002 we reported on a protocol for dividing the frontal lobe into major components and demonstrated its feasibility by applying the technique to MRI (magnetic resonance imaging) data acquired from boys with ADHD or Tourette syndrome, whose scans were compared to those acquired from a group of typically developing (TD) controls (Kates et al., 2002b). Our functionally oriented 2002 method divided the frontal lobe into five modules: primary motor cortex, anterior cingulate, premotor cortex, prefrontal cortex (PFC) and deep white matter (DWM) (Kates et al., 2002b). Instead of relying only on prominent gyri and sulci, this protocol 
utilized both major and minor easily identifiable anatomical landmarks along with cut planes along the trajectories of sulci or at sulcal intersections and turning points. The definition of deep white matter was novel and allowed a distinction between short association fibers (gyral white matter) and long or projecting association fibers (DWM), (Makris et al., 1999) increasing the relevance of regional measurements.

In this paper we present the second stage in the development of this technique. As in the first stage of the project, frontal lobe sub-regions were delineated on the bases of consistent sulcal/ gyral landmarks chosen because of their relationships to functional boundaries indicated by cytoarchitectonic, electrophysiological, magnetic stimulation and functional imaging findings (Geschwind, 1979; Zilles et al., 1990; Geyer et al., 2000; Tekin et al., 2002). To balance the competing priorities of validity and reliability, sulcal contours were used when appropriate for defining a functional area and cut planes were used where functional and sulcal divisions diverged or when the level of anatomical detail in MR images or degree of inter-subject variability made the use of standard anatomical landmarks excessively difficult. This strategy led to high protocol reliability despite significant inter-subject cortical variability (Ono et al., 1990; Zilles et al., 1997). On the foundations of traditional and more recent anatomical and functional literature (McGlinchey-Berroth et al., 1995; Tekin et al., 2002; Ongur et al., 2003; Costafreda et al., 2006; Rypma, 2006; Mostofsky et al., 2007; Rushworth et al., 2007) the premotor and prefrontal modules were divided into several anatomo-physiologic sub-regions. The premotor module was subdivided into supplementary motor complex (SMC), frontal eye field (FEF) and lateral premotor cortex (LPM). The PFC module was subdivided into medial PFC, dorsolateral PFC (DLPFC), inferior PFC, lateral orbitofrontal cortex (OFC) and medial OFC. The SMC and FEF are traditionally described by physiological approaches, so the proposed definitions can be considered as delineating anatomical-equivalent SMC and anatomical FEF regions. The SMC includes the supplementary motor area (SMA), the preSMA and the supplementary eye field. Similar approaches to studying selective frontal regions provide additional support to the overall strategy of the present study (Howard et al., 2003; Lacerda et al., 2003).

The labor intensive nature of manual delineation methods provide irreplaceable data on subjects' individual anatomy compared to automatic or semi-automatic methods that have also been applied to frontal assessments (Mostofsky et al., 2002; Carter et al., 2008a; Carter et al., $2008 b$ ). This attention to individual differences in sulcal patterns is particularly important for studying disorders with subtle neuroanatomical abnormalities, such as ADHD and learning disabilities, where small changes spanning a functional region are often predicted (Kelly et al., 2007).

As proof-of-principle, and in line with the implementation of the first stage of this protocol (Kates et al., 2002b), the present, more comprehensive protocol was applied to MR data acquired from a group of boys with ADHD and compared to that of matched TD controls. Frontal abnormalities have been widely reported in ADHD but the relative contributions of different frontal regions remain poorly defined (Castellanos et al., 1996; Castellanos et al., 2002; Kates et al., 2002b; Mostofsky et al., 2002; Sowell et al., 2003; Shaw et al., 2006; Shaw et al., 2007). The application of our frontal lobe parcellation technique has the potential to illuminate the roles of discrete functionally relevant regions in ADHD and other neuropsychological disorders.

\section{2.) METHODS}

\section{1) Participants}

2.1.1.) Group comparison-The present frontal lobe parcellation method was applied to brain MR data acquired from 15 boys with ADHD and from 15 TD control boys, 8-12 years 
old. The two groups were closely matched on age (ADHD mean age $=10.5$ years, Control mean age $=11.0$ years, $P=0.28, d f=1)$ and full scale IQ (ADHD mean FSIQ $=114.5$, Control mean FSIQ $=117.9, P=0.64, d f=1$ ), based on one-way analysis of variance (ANOVA) calculations. The group was restricted to boys for this initial study because regional brain volume differences in ADHD have primarily been found only in boys (Castellanos et al., 2001).

2.1.2.) Reliability analysis-A subset of the group was used to establish intra- and interrater reliability for the parcellation protocol. For the first step, the isolation of the frontal lobe, the reliability subgroup consisted of 5 ADHD boys and 5 TD boys, matched on age and FSIQ. For reliability on the frontal sub-parcellation, the reliability group consisted of 5 boys, 2 ADHD and $3 \mathrm{TD}$, randomly chosen from the isolation reliability group. This produced a total of 10 parcellated hemispheres, 5 left and 5 right, which were analyzed independently.

2.1.3.) Participant screening-Participants were recruited from outpatient clinics at the Kennedy Krieger Institute and from local pediatricians, chapters of Children and Adults with Attention-Deficit/Hyperactivity Disorder (CHADD), schools, social/service organizations (e.g., Boy Scouts), and advertisements in the community (e.g., postings at libraries).

All children entering the study had a FSIQ of 80 or higher based on performance on the Wechsler Intellectual Scale for Children WISC- 3rd edition (Wechsler, 1991) $(n=5)$ or the WISC-4th edition (Wechsler, 2003) $(n=25)$. None of the children had a history of speech/ language disorder or a reading disability, and all had a basic reading standard score of 85 (16 ${ }^{\text {th }}$ percentile) or higher on the word reading subtest from the Wechsler Individual Achievement Test, 1st Edition (WIAT) (Wechsler, 1992) $(n=4)$ or Second Edition (WIATII) (Wechsler, 2002) $(n=26)$.

The structured parent interview, Diagnostic Interview for Children and Adolescents- 4th edition (DICA-IV) (Reich et al., 1997) and ADHD-specific and broad behavior rating scales (Conners' Parent and Teacher Rating Scales-Revised CPRS-R, CTRS-R, long form (Conners, 1997)]) were used to confirm diagnosis. Control children with T-scores greater than 60 on the ADHD (DSM-IV Inattention, DSM-IV Hyperactivity) subscales of CPRS-R were excluded from the study. The CPRS-R and DSM IV criteria were used to evaluate ADHD subtype (Predominantly Hyperactive-Impulsive, Predominantly Inattentive or Combined). Of the 15 ADHD subjects analyzed, 7 were Combined subtype and 8 were Predominantly Inattentive subtype.

The DICA-IV was also used to examine for the presence of other psychiatric disorders. Any children, whether ADHD or Control, with co-morbid conduct disorder, mood disorder, generalized anxiety disorder, separation anxiety disorder, or obsessive-compulsive disorder were excluded from this study. ADHD children with Oppositional Defiant Disorder (ODD) and/or simple phobia were included; ODD was present in 5 of the ADHD subjects and simple phobia was present in 3 , including 1 subject with both.

Children with ADHD were also excluded from the study if they were taking long-acting psychoactive medications. Additional exclusion criteria for controls included history of mental health service utilization for behavioral or emotional problems, history of academic problems requiring school-based intervention services or history of defined primary reading or languagebased learning disability.

The study was approved by the Johns Hopkins University School of Medicine Institutional Review Board. Participants provided written consent (caregivers) and assent (children) and received a copy of the consent form before beginning testing. 


\section{2.) MRI image acquisition and preprocessing}

T1-weighted 3D-volume MPRAGE images of the brain (matrix size $=256 \times 256$, echo time $=3 \mathrm{~ms}$, repetition time $=7 \mathrm{~ms}$, field of view $=260 \mathrm{~mm}$, slice thickness $=1.2 \mathrm{~mm}$ ) were acquired using a 1.5T Philips Gyroscan NT (Royal Philips Electronics, Amsterdam, The Netherlands) for each subject in the study. All image processing was conducted using the MIPAV (Medical Image Processing, Analysis and Visualization) software package from the Center for Imaging Technology (CIT) of the National Institutes of Health (NIH, Bethesda, MD, USA) (McAuliffe et al., 2001). Initial post-acquisition processing consisted of intensity normalization, manual removal of skull, membrane and vascular structures and Anterior Commissure-Posterior Commissure (AC-PC) alignment. The AC-PC alignment created a coordinate system in which one axis is defined by the line between the $\mathrm{AC}$ and $\mathrm{PC}$, the second is orthogonal to the $\mathrm{AC}-\mathrm{PC}$ line and passes through the inter-hemispheric fissure, and the third is orthogonal to both of the previous two.

\section{3.) Parcellation method}

In this protocol, the frontal lobe is manually isolated from the rest of the brain and subdivided into 11 sub-regions in each hemisphere using gyral and sulcal landmarks. A sample final parcellation is shown in Figures 1 and 2 and the protocol is described in full detail in the supplementary material. A summary of the main boundaries and individual functions of the frontal lobe sub-regions in list form is provided in Supplementary Table 1. Throughout the protocol, landmarks were chosen for their use in delineating functional sub-divisions of the frontal lobe, based on multiple cytoarchitectonic, electrophysiological, magnetic stimulation and functional imaging findings referenced fully in the supplementary material. The AC-PC alignment creates a coordinate system in which individual brains can be placed in a standard orientation. Sulcal localization and patterns are based on reviews by Ono et al. (Ono et al., 1990) and Zilles (Zilles et al., 1997) and on our experiences with the initial implementation of this approach (Aylward et al., 1997; Kates et al., 2002b).

\section{4.) Segmentation}

Segmentation into gray matter (GM), white matter (WM) and cerebrospinal fluid (CSF) tissue components was carried out in MIPAV using a fuzzy segmentation algorithm (FANTASM) (McAuliffe et al., 2001). Images were normalized to remove inhomogeneity artifacts, diffused to improve signal-to-noise ratio and rotated to match the AC-PC aligned orientation of the parcellated regions before segmentation. This segmentation method produces three images, one each for the GM, WM and CSF components, with voxel intensity values ranging from zero to one, indicating the probability that an individual voxel belongs to each category. This value also defines the proportion of each voxel that should be assigned to each tissue class.

\section{5.) Volume calculation}

At the completion of the frontal lobe parcellation, each voxel of the frontal lobe is assigned to a single frontal sub-region. Each region can be defined by a volume of interest (VOI) file that can be used to calculate image statistics within the VOI when applied to an underlying image. For each region, the number of voxels was measured and multiplied by the volume of a single voxel to obtain the total volume contained in the VOI. To determine GM and WM volumes the VOI was applied to the GM or WM segmentation image and the number of voxels and average intensity of voxels within the VOI were calculated. The final GM or WM volume was obtained by multiplying the number of voxels within the VOI by the average intensity of the all voxels within the VOI and by the volume of a single voxel. 


\section{6.) Statistical analyses}

2.6.1.) Reliability analysis-In the reliability analysis, the frontal lobe isolation and parcellation components of the protocol were examined separately. For the frontal lobe isolation reliability component, the volumes of the isolated frontal lobes of 5 boys with ADHD and 5 TD controls were measured. For the frontal lobe sub-parcellation component, the volumes of left and right hemisphere frontal sub-regions were measured for 5 boys, 2 ADHD and 3 TD. The analyses of left and right hemisphere sub-regions were carried out independently. Raters were blind to diagnosis. When using interclass correlation coefficients to determine reliability, coefficients of 0.8 and greater are generally considered to be indicative of high reliability having been achieved while a value between 0.7 and 0.8 is considered to be an acceptable result (Shrout \& Fleiss, 1979; Kates et al., 1997).

A single rater (MER) completed the isolation and parcellation steps twice, using the second set of isolated frontal lobes as the starting point for both parcellation attempts. The time elapsed between the two measurements for each region was at least two weeks. Comparing unsegmented, absolute volume measurements for each subregion in each hemisphere, 19 out of 22 intra-rater intraclass correlation coefficients (ICC) were 0.9 or greater. Full results are shown in Table 1.

A second rater (DC) independently isolated the frontal lobes of the same 10 boys and the results were compared to the isolations by the first rater. Following this, and beginning with the first rater's isolated frontal lobes, the second rater carried out the parcellation of all frontal lobes in the 5 boys in the parcellation reliability group. The volumes of the parcellated regions produced by the second rater were compared to those of the first rater. Inter-rater ICC for the 22 frontal sub-regions ranged from 0.724 to 0.997 . Full results are shown in Table 1.

2.6.2.) Group comparison-For the group comparison study, the complete parcellation protocol was applied to the brain MR data of 15 boys with ADHD and 15 TD control boys. Mann-Whitney tests were used to examine the effect of diagnosis (ADHD vs. Control) on total, gray and white matter volume in each frontal lobe region. A statistical threshold of $P<0.05$ was used to determine significance and the SPSS 15.0 statistical analysis package (SPSS, Inc., Chicago, IL, USA) was used to carry out all analyses.

Despite the possibility of false negative findings, non-parametric Mann-Whitney tests were chosen for analysis of group differences because of the relatively small number of subjects in each group and non-normal distribution of many of the volume measures. Results were corrected for multiple comparisons using the Bonferroni approach with a hierarchical anatomybased strategy for the 11 sub-regions in each hemisphere (Carter et al., 2008a; Carter et al., 2008b). On the basis of previous findings (Kates et al., 2002b; Mostofsky et al., 2002) differences in prefrontal and premotor regions were hypothesized, however the specific subregions that would be affected were difficult to predict. Therefore, we chose to report both uncorrected and Bonferroni corrected results for all regions. Uncorrected $P$-values are reported and significant corrected $P$-values are noted (in Tables 2 and 3).

Total cerebral volume (TCV) for each subject was calculated using Freesurfer, an automated surface-based analysis program (http://www.nmr.mgh.harvard.edu, Martinos Center for Biomedical Imaging, Boston, MA, USA). As reported in Wolosin et al. (Wolosin et al., 2007) in which the same measurement method was used, the ADHD boys showed decreased TCV compared to TD control boys (ADHD, $1174 \pm 77 \mathrm{~cm}^{3}$; Control $1246 \pm 67 \mathrm{~cm}^{3}, P=0.024$ ) in a Mann-Whitney comparison. Given this difference, all frontal lobe regions were examined both before and after normalizing for TCV, with the results for normalized volumes considered the main analysis. Mann-Whiney tests were conducted on the normalized volumes in order to investigate relative volume differences; the tests were repeated for original, non-normalized 
values, allowing for an examination of absolute volumes as well. Normalization was accomplished by multiplying the original measured volume by the average TCV of the entire analysis group and dividing by the individual subject's TCV.

Group mean volumes, standard deviations and significance results for the left and right hemispheres are reported in Tables 2 and 3 respectively.

\section{3.) RESULTS}

\section{1.) Reliability}

ICC calculations using un-segmented, absolute parcellation volumes were used to determine intra- and inter-rater reliability coefficients for the frontal lobe and all subregions. Intra- and inter-rater reliability coefficients for the initial, full frontal lobe delineation were 0.998 and 0.997 respectively. Intra-rater coefficients for the 22 frontal sub-regions (11 in each hemisphere) ranged from 0.778 to 0.997 , with all but three greater than 0.9 , and all but one greater than 0.8. Inter-rater sub-region coefficients ranged from 0.724 to 0.997 , with all but six measures above 0.9 and all but two above 0.8 , indicating a high degree of reliability. For one region, the R FEF, one subject was dropped from the ICC calculations due to an unusual sulcal configuration. Although not ideal, ICC coefficients below 0.8 are not unexpected for small regions with multiple boundaries. Full reliability results are presented in Table 1.

\section{2.) Protocol implementation - group difference analysis}

Mann-Whitney tests were used to examine group differences (ADHD vs. Control) in total, GM and $\mathrm{WM}$ volumes in each frontal lobe and parcellated sub-region before and after normalization for TCV.

At the cerebral level, boys with ADHD showed reductions of $6.1 \%, 5.5 \%$ and $5.8 \%$ in left, right and combined TCV respectively (all $P=0.024$ ) in Mann-Whitney tests. Similar reductions were seen in the ADHD group in the absolute left frontal lobe total $(5.6 \%, P=0.040)$ and GM volumes $(6.0 \%, P=0.021)$ before normalization for TCV. After normalization, the left frontal lobe volume reductions were no longer significant. No significant differences between diagnosis groups were found in the right frontal lobe volume. Further, no significant differences between groups in right hemisphere frontal lobe sub-regions volumes were found; all significant difference results were in the left hemisphere alone.

In the left SMC, boys with ADHD showed a reduction in normalized WM volume $(P=0.014)$. Before normalization for TCV, reductions in left SMC absolute total volume $(P=0.029)$ and absolute WM volume $(P=0.005)$ were significant.

Boys with ADHD showed a reduction in normalized total $(P=0.014)$ and WM volumes $(P=$ $0.004)$ in the left DLPFC. Before normalization, reductions in the left DLPFC total, gray and white matter volumes $(P=0.003, P=0.005, P=0.007)$ were significant. After correction for multiple comparisons, the reductions in normalized left DLPFC WM volume $(P$-corrected $=$ $0.049)$ and absolute left DLPFC volume $(P$-corrected $=0.033)$ remained significant.

In the left medial PFC, normalization for TCV resulted in non-significant group differences. Before normalization, significant differences in total, gray and white matter volumes $(P=$ $0.014, P=0.024, P=0.017$ ) were found, with the ADHD group having reduced volumes in all tissue categories.

A less robust, but statistically significant result was found in the left FEF, where boys with ADHD showed increased absolute left FEF GM volume $(P=0.049)$. The difference was not significant for normalized volumes. 
Group mean volumes, standard deviations and significance results for the left and right hemispheres are reported in Tables 2 and 3 respectively. Plots of normalized individual subject volumes for the left DLPFC, left SMC WM and left DLPFC WM are shown in Figure 3.

\section{4.) DISCUSSION}

We have developed a highly reliable method to manually parcellate brain MR data into functionally relevant frontal lobe sub-regions. The protocol expands upon previous approaches by focusing on divisions that define functionally distinct frontal components, rather than primarily or predominantly anatomical components, as in previously published methodologies (Buchanan et al., 1998; Kennedy et al., 1998; Convit et al., 2001; Howard et al., 2003). The present approach builds on our 2002 publication, in which we presented a method for parcellating the frontal lobe into five major functional components (Kates et al., 2002b). In the present protocol the prefrontal and premotor regions are sub-divided into functionally relevant sub-regions. These functional divisions allow for more detailed examinations of the pathophysiology of disorders, such as ADHD, in which behavioral deficits and previous findings predict selective frontal lobe involvement. The definition of functionally relevant subregions also allows for the investigation of direct correlations between anatomical abnormalities and both functional imaging features and behavioral characteristics. The initial application of the protocol to a cohort of boys with ADHD, who generally display relatively small abnormalities in frontal volume (Kelly et al., 2007), provides evidence of the feasibility and sensitivity of the method.

When applied to a group of 15 boys with ADHD and 15 age- and IQ- matched controls, the methods identified significant reductions in bilateral cerebral volume and left frontal lobe volume along with highly significant total and segmented volume reductions in the SMC, medial PFC and DLPFC sub-regions of the left frontal lobe. Significant differences in relative, normalized for TCV volumes were isolated to the SMC WM component and the DLPFC total and WM volumes. The fact that no significant differences were found in the right hemisphere is inconsistent with some evidence supporting the hypothesis of right hemisphere dominance in attention, which also includes frontal "intentional" processes critical to control of off-task impulsive and hyperactive behavior (Heilman, 1991; Petit et al., 2007), but is in agreement with many studies of ADHD that have found abnormalities in the left frontal lobe, including reduced volume in left premotor and prefrontal cortices, localized reductions in inferior dorsal prefrontal areas, thinning in the medial PFC and delayed attainment of peak cortical thickness (Castellanos et al., 2002; Kates et al., 2002b; Mostofsky et al., 2002; Sowell et al., 2003; Shaw et al., 2006; Shaw et al., 2007). It is possible that more bilateral effects of diagnosis will be seen when a larger number of subjects have been analyzed, including a larger percentage of subjects with Hyperactive-Impulsive and Combined ADHD subtype. On the other hand, absence of volumetric differences in the right frontal lobe does not exclude functional abnormalities in this frontal component.

The left hemisphere frontal lobe findings are consistent with general theories of ADHD that implicate dysfunction in frontal regions and circuits that support executive function. For the SMC and DLPFC in particular, precise associations can be made between core observed characteristics of ADHD and the known functional specialization of those regions. The SMC, particularly its rostral, "pre-SMA" portion is known to be important in the planning and selection of motor responses, with response inhibition seen as a specific sub-function of this role (Mostofsky et al., 2003; Isoda \& Hikosaka, 2007; Picton et al., 2007; Rushworth et al., 2007; Simmonds et al., 2007; Mostofsky \& Simmonds, 2008; Simmonds et al., 2008). There is a good deal of evidence showing that children with ADHD perform inconsistently or at an impaired level on a range of impulse control tasks (e.g., (Wodka et al., 2007)) and that ADHD is associated with structural (Shaw et al., 2006) and functional (Suskauer et al., 2008) 
abnormalities in the region of the SMC. The DLPFC has a known role in working memory and other aspects of executive function (Braver et al., 1997; DEsposito et al., 2000; Alvarez et al., 2006) and strong evidence exists for deficits in working memory in ADHD (Martinussen et al., 2005; Willcutt et al., 2005; Rapport et al., 2008). In this context, the decreases in left hemisphere SMC and DLPFC volumes found in our ADHD group are the anatomical correlates of executive function deficits that are chief characteristics of ADHD. The volume reductions in the SMC and DLPFC appear to be preferentially due to decreases in their white matter components, suggesting a primarily axonal abnormality in the ADHD group. Further, the results imply a selective connectivity problem, affecting short cortico-cortical connections while sparing the longer, projecting (cortico-cortical and cortico-subcortical) association fibers represented by the DWM, where no volume reductions were detected.

The results of this ADHD vs. Control comparison study should be regarded as preliminary until the study is expanded to a larger group of subjects, including girls with ADHD. Regional brain volume differences in ADHD have primarily been found only in boys (Castellanos et al., 2001) but our subdivision of the frontal lobe may reveal more localized, function-specific abnormalities in a female population. A larger number of subjects would provide greater power for detecting differences as well as a more inclusive sample of sulcal variability and ADHD phenotype. Additionally, the use of stringent, non-parametric Mann-Whitney tests for statistical analysis may have reduced the possibility of detecting group differences in this study; additional analyses will be required for a complete assessment of the protocol's sensitivity.

The theoretical foundations for our protocol include the sometimes divergent relationships between gyral/sulcal landmarks and cytoarchitectonical and functional organization of the cortex (Zilles et al., 1990; Zilles et al., 1997; Geyer et al., 2000; Ongur et al., 2003) and the increasing body of information on the functional relevance of discrete frontal sub-regions, particularly within the prefrontal and premotor regions (Tekin et al., 2002; Graziano et al., 2007; Mostofsky et al., 2007; Rushworth et al., 2007). Nonetheless, as in other recent protocols (Howard et al., 2003; Lacerda et al., 2003), adaptations were made in order to increase reliability. The use of cut planes to define regional boundaries where cortical complexity or inter-subject sulcal variability make more detailed definitions excessively difficult is the primary limitation of our parcellation method, though an approach employed in previous protocols (Kennedy et al., 1998; Crespo-Facorro et al., 1999). The need for a large time commitment and raters with a fairly sophisticated knowledge of neuroanatomy make this protocol more difficult to apply than automated or semi-automated parcellation methods. However, the functional validity of the resultant sub-divisions is a major improvement over faster, easier techniques. With divisions corresponding to functional regions, the parcellation method presented here has the potential to further our understanding of the anatomic substrates of the large number of disorders in which frontal lobe abnormalities are thought to be a contributing factor.

\section{Supplementary Material}

Refer to Web version on PubMed Central for supplementary material.

\section{Acknowledgments}

We are grateful to the families that participated in our studies of ADHD.

Funding: National Institutes of Health (K02 NS044850 and R01_NS048527 to S.H.M., P30 HD-24061 to M.A.K \& W.E.K.), The Charles Dana Foundation (W.E.K.) 


\section{References}

Acosta MT, Pearl PL. Imaging data in autism: from structure to malfunction. Seminars in Pediatric Neurology 2004;11:205-213. [PubMed: 15575415]

Alvarez JA, Emory E. Executive Function and the Frontal Lobes: A Meta-Analytic Review. Neuropsychology Review. 2006

Ashe J, Lungu OV, Basford AT, Lu X. Cortical control of motor sequences. Current Opinion in Neurobiology 2006;16:213-221. [PubMed: 16563734]

Aylward EH, Augustine A, Li Q, Barta PE, Pearlson GD. Measurement of frontal lobe volume on magnetic resonance imaging scans. Psychiatry Research 1997;75:23-30. [PubMed: 9287371]

Braver TS, Cohen JD, Nystrom LE, Jonides J, Smith E, Noll DC. A parametric study of prefrontal cortex involvement in human working memory. Neuroimage 1997;5:49-62. [PubMed: 9038284]

Buchanan RW, Vladar K, Barta PE, Pearlson GD. Structural evaluation of the prefrontal cortex in schizophrenia. American Journal of Psychiatry 1998;155:1049-1055. [PubMed: 9699693]

Carter JC, Capone GT, Kaufmann WE. Neuroanatomic correlates of autism and stereotypy in children with Down syndrome. Neuroreport 2008a;19:653-656. [PubMed: 18382280]

Carter JC, Lanham DC, Pham D, Bibat G, Naidu S, Kaufmann WE. Selective cerebral volume reduction in Rett syndrome: a multiple-approach MR imaging study. American Journal of Neuroradiology 2008b;29:436-441. [PubMed: 18065507]

Castellanos FX, Geidd JN, Marsh WL, Hamburger SD, Vaituzis AC, Dickstein DP, Sarfatti SE, Vauss YC, Snell JW, Lange N, Kaysen D, Krain AL, Ritchie GF, Rajapakse JC, Rapoport JL. Quantitative brain magnetic resonance imaging in attention-deficit hyperactivity disorder. Archives of General Psychiatry 1996;53:607-616. [PubMed: 8660127]

Castellanos FX, Giedd JN, Berquin PC, Walter JM, Sharp W, Tran T, Vaituzis AC, Blumenthal JD, Nelson J, Bastain TM, Zijdenbos A, Evans AC, Rapoport JL. Quantitative brain magnetic resonance imaging in girls with attention-deficit/hyperactivity disorder. Archives of General Psychiatry 2001;58:289-295. [PubMed: 11231836]

Castellanos FX, Lee PP, Sharp W, Jeffries NO, Greenstein DK, Clasen LS, Blumenthal JD, James RS, Ebens CL, Walter JM, Zijdenbos A, Evans A, Giedd JN, Rapoport JL. Developmental trajectories of brain volume abnormalities in children and adolescents with attention-deficit/hyperactivity disorder. Journal of the American Medical Association 2002;288:1740-1748. [PubMed: 12365958]

Conners, CK. Conners' Rating Scales - Revised. Multi-Health Systems, Inc; North Tonawanda, New York: 1997.

Convit A, Wolf OT, de Leon MJ, Patalinjug M, Kandil E, Caraos C, Scherer A, Saint Louis LA, Cancro R. Volumetric analysis of the pre-frontal regions: findings in aging and schizophrenia. Psychiatry Research 2001;107:61-73. [PubMed: 11530273]

Costafreda SG, Fu CH, Lee L, Everitt B, Brammer MJ, David AS. A systematic review and quantitative appraisal of fMRI studies of verbal fluency: role of the left inferior frontal gyrus. Human Brain Mapping 2006;27:799-810. [PubMed: 16511886]

Courchesne E, Pierce K, Schumann CM, Redcay E, Buckwalter JA, Kennedy DP, Morgan J. Mapping early brain development in autism. Neuron 2007;56:399-413. [PubMed: 17964254]

Crespo-Facorro B, Kim JJ, Andreasen NC, O’Leary DS, Wiser AK, Bailey JM, Harris G, Magnotta VA. Human frontal cortex: An MRI-based parcellation method. Neuroimage 1999;10:500-519. [PubMed: 10547328]

Cutting LE, Huang GH, Zeger S, Koth CW, Thompson RE, Denckl MB. Growth curve analyses of neuropsychological profiles in children with neurofibromatosis type 1: specific cognitive tests remain "spared" and "impaired" over time. Journal of the International Neuropsychological Society 2002;8:838-846. [PubMed: 12240748]

Desikan RS, Segonne F, Fischl B, Quinn BT, Dickerson BC, Blacker D, Buckner RL, Dale AM, Maguire RP, Hyman BT, Albert MS, Killiany RJ. An automated labeling system for subdividing the human cerebral cortex on MRI scans into gyral based regions of interest. Neuroimage 2006;31:968-980. [PubMed: 16530430]

DEsposito M, Postle BR, Rypma B. Prefrontal cortical contributions to working memory: evidence from event-related fMRI studies. Experimental Brain Research 2000;133:3-11. 
Fredericksen KA, Cutting LE, Kates WR, Mostofsky SH, Singer HS, Cooper KL, Lanham DC, Denckla MB, Kaufmann WE. Disproportionate increases of white matter in right frontal lobe in Tourette syndrome. Neurology 2002;58:85-89. [PubMed: 11781410]

Fuster, JM. The prefrontal cortex. Raven Press; New York: 1997.

Geschwind N. Specializations of the human brain. Scientific American 1979;241:180-199. [PubMed: 493918]

Geyer S, Matelli M, Luppino G, Zilles K. Functional neuroanatomy of the primate isocortical motor system. Anatomy and Embryology (Berlin) 2000;202:443-474.

Gothelf D, Furfaro JA, Hoeft F, Eckert MA, Hall SS, O'Hara R, Erba HW, Ringel J, Hayashi KM, Patnaik S, Golianu B, Kraemer HC, Thompson PM, Piven J, Reiss AL. Neuroanatomy of fragile X syndrome is associated with aberrant behavior and the fragile $\mathrm{X}$ mental retardation protein (FMRP). Annals of Neurology 2008;63:40-51. [PubMed: 17932962]

Graziano MS, Aflalo TN. Mapping behavioral repertoire onto the cortex. Neuron 2007;56:239-251. [PubMed: 17964243]

Greenwood RS, Tupler LA, Whitt JK, Buu A, Dombeck CB, Harp AG, Payne ME, Eastwood JD, Krishnan KR, MacFall JR. Brain morphometry, T2-weighted hyperintensities, and IQ in children with neurofibromatosis type 1. Archives of Neurology 2005;62:1904-1908. [PubMed: 16344348]

Heilman KM. A possible pathophysiologic substrate of Attention Deficit Hyperactivity Disorder. Journal of Child Neurology 1991;6(S1):S74-S79.

Howard MA, Roberts N, Garcia-Finana M, Cowell PE. Volume estimation of prefrontal cortical subfields using MRI and stereology. Brain Research Protocols 2003;10:125-138. [PubMed: 12565683]

Isoda M, Hikosaka O. Switching from automatic to controlled action by monkey medial frontal cortex. Nature Neuroscience 2007;10:240-248.

John JP, Wang L, Moffitt AJ, Singh HK, Gado MH, Csernansky JG. Inter-rater reliability of manual segmentation of the superior, inferior and middle frontal gyri. Psychiatry Research 2006;148:151163. [PubMed: 17088050]

Kates WR, Abrams MT, Kaufmann WE, Breiter SN, Reiss AL. Reliability and validity of MRI measurement of the amygdala and hippocampus in children with fragile X syndrome. Psychiatry Research 1997;75:31-48. [PubMed: 9287372]

Kates WR, Folley BS, Lanham DC, Capone GT, Kaufmann WE. Cerebral growth in Fragile X syndrome: review and comparison with Down syndrome. Microscopical Research and Technology 2002a; 57:159-167.

Kates WR, Frederikse M, Mostofsky SH, Folley BS, Cooper K, Mazur-Hopkins P, Kofman O, Singer HS, Denckla MB, Pearlson GD, Kaufmann WE. MRI parcellation of the frontal lobe in boys with attention deficit hyperactivity disorder or Tourette syndrome. Psychiatry Research 2002b;116:6381. [PubMed: 12426035]

Kelly AM, Margulies DS, Castellanos FX. Recent advances in structural and functional brain imaging studies of attention-deficit/hyperactivity disorder. Current Psychiatry Reports 2007;9:401-407. [PubMed: 17915080]

Kennedy DN, Lange N, Makris N, Bates J, Meyer J, Caviness V. Gyri of the human neocortex: An MRIbased analysis of volume and variance. Cerebral Cortex 1998;8:372-384. [PubMed: 9651132]

Lacerda AL, Hardan AY, Yorbik O, Keshavan MS. Measurement of the orbitofrontal cortex: a validation study of a new method. Neuroimage 2003;19:665-673. [PubMed: 12880797]

Makris N, Meyer JW, Bates JF, Yeterian EH, Kennedy DN, Caviness VS. MRI-Based topographic parcellation of human cerebral white matter and nuclei II. Rationale and applications with systematics of cerebral connectivity. Neuroimage 1999;9:18-45. [PubMed: 9918726]

Marsh R, Zhu H, Wang Z, Skudlarski P, Peterson BS. A developmental fMRI study of self-regulatory control in Tourette's syndrome. American Journal of Psychiatry 2007;164:955-966. [PubMed: 17541057]

Martinussen R, Hayden J, Hogg-Johnson S, Tannock R. A meta-analysis of working memory impairments in children with attention-deficit/hyperactivity disorder. Journal of the American Academy of Child and Adolescent Psychiatry 2005;44:377-384. [PubMed: 15782085] 
McAuliffe M, Lalonde E, McGarry D, Gandler W, Csaky K, Trus B. Medical image processing, analysis and visualization in clinical research. IEEE Symposium on computer-based medical systems 2001:381-386.

McGlinchey-Berroth R, Cermak LS, Carrillo MC, Armfield S, Gabrieli JDE, Disterhoft JF. Impaired delay eyeblink conditioning in amnesic Korsakoff's patients and recovered alcoholics. Alcoholism: Clinical and Experimental Research 1995;19:1127-1132.

Mostofsky SH, Burgess MP, Gidley Larson JC. Increased motor cortex white matter volume predicts motor impairment in autism. Brain 2007;130:2117-2122. [PubMed: 17575280]

Mostofsky SH, Cooper KL, Kates WR, Denckla MB, Kaufmann WE. Smaller prefrontal and premotor volumes in boys with ADHD. Biological Psychiatry 2002;52:785-794. [PubMed: 12372650]

Mostofsky SH, Schafer JGB, Abrams MT, Goldberg MC, Flower AA, Courtney SM, Calhoun VD, Kraut MA, Denckla MB, Pekar JJ. FMRI evidence that the neural basis of response inhibition is taskdependent. Cognitive Brain Research 2003;17:419-430. [PubMed: 12880912]

Mostofsky SH, Simmonds DJ. Response inhibition and response selection: two sides of the same coin. Journal of Cognitive Neuroscience 2008;20:751-761. [PubMed: 18201122]

Nigg JT, Casey BJ. An integrative theory of attention-deficit/hyperactivity disorder based on the cognitive and affective neurosciences. Development and Psychopathology 2005;17:785-806. [PubMed: 16262992]

Ongur D, Ferry AT, Price JL. Architectonic subdivision of the human orbital and medial prefrontal cortex. The Journal of Comparative Neurology 2003;460:425-449. [PubMed: 12692859]

Ono, M.; Kubik, S.; Abernathey, C. Atlas of the Cerebral Sulci. Thieme Medical Publishers; New York: 1990.

Petit L, Simon G, Joliot M, Andersson F, Bertin T, Zago L, Mellet E, Tzourio-Mazoyer N. Right hemisphere dominance for auditory attention and its modulation by eye position: an event related fMRI study. Restorative Neurology and Neuroscience 2007;25:211-225. [PubMed: 17943000]

Picton TW, Stuss DT, Alexander MP, Shallice T, Binns MA, Gillingham S. Effects of focal frontal lesions on response inhibition. Cerebral Cortex 2007;17:826-838. [PubMed: 16699079]

Pinter JD, Eliez S, Schmitt JE, Capone GT, Reiss AL. Neuroanatomy of Down's syndrome: a highresolution MRI study. American Journal of Psychiatry 2001;158:1659-1665. [PubMed: 11578999]

Porter MA, Coltheart M, Langdon R. The neuropsychological basis of hypersociability in Williams and Down syndrome. Neuropsychologia 2007;45:2839-2849. [PubMed: 17597166]

Rademacher J, Galaburda AM, Kennedy DN, Filipek PA, Caviness VS. Human cerebral cortex: localization, parcellation, and morphometry with magnetic resonance imaging. Journal of Cognitive Neuroscience 1992;4:352-374.

Rapport MD, Alderson RM, Kofler MJ, Sarver DE, Bolden J, Sims V. Working memory deficits in boys with attention-deficit/hyperactivity disorder (ADHD): the contribution of central executive and subsystem processes. Journal of Abnormal Child Psychology 2008;36:825-837. [PubMed: 18317920]

Reich, W.; Welner, Z.; Herjanic, B. The Diagnostic Interview for Children and Adolescents-IV. MultiHealth Systems, Inc; North Tonowanda, New York: 1997.

Reiss AL, Eckert MA, Rose FE, Karchemskiy A, Kesler S, Chang M, Reynolds MF, Kwon H, Galaburda A. An experiment of nature: brain anatomy parallels cognition and behavior in Williams syndrome. Journal of Neuroscience 2004;24:5009-5015. [PubMed: 15163693]

Rushworth MF, Buckley MJ, Behrens TE, Walton ME, Bannerman DM. Functional organization of the medial frontal cortex. Current Opinion in Neurobiology 2007;17:220-227. [PubMed: 17350820]

Rypma B. Factors controlling neural activity during delayed-response task performance: testing a memory organization hypothesis of prefrontal function. Neuroscience 2006;139:223-235. [PubMed: 16343777]

Sanides, F. Representation in the cerebral cortex and its areal lamination patterns. In: Bourne, GF., editor. The structure and function of nervous tissue. Academic Press; New York, NY: 1972. p. 330-453.

Shad MU, Muddasani S, Keshavan MS. Prefrontal subregions and dimensions of insight in first-episode schizophrenia--a pilot study. Psychiatry Research 2006;146:35-42. [PubMed: 16361089] 
Shaw P, Eckstrand K, Sharp W, Blumenthal J, Lerch JP, Greenstein D, Clasen L, Evans A, Giedd J, Rapoport JL. Attention-deficit/hyperactivity disorder is characterized by a delay in cortical maturation. The Proceedings of the National Academy of Sciences (USA) 2007;104:19649-19654.

Shaw P, Lerch J, Greenstein D, Sharp W, Clasen L, Evans A, Giedd J, Castellanos FX, Rapoport J. Longitudinal mapping of cortical thickness and clinical outcome in children and adolescents with attention-deficit/hyperactivity disorder. Archives of General Psychiatry 2006;63:540-549. [PubMed: 16651511]

Shrout PE, Fleiss JL. Intraclass Correlations: Uses in Assessing Rater Reliability. Psychological Bulletin 1979;2:420-428. [PubMed: 18839484]

Simmonds DJ, Fotedar SG, Suskauer SJ, Pekar JJ, Denckla MB, Mostofsky SH. Functional brain correlates of response time variability in children. Neuropsychologia 2007;45:2147-2157. [PubMed: 17350054]

Simmonds DJ, Pekar JJ, Mostofsky SH. Meta-analysis of Go/No-go tasks demonstrating that fMRI activation associated with response inhibition is task-dependent. Neuropsychologia 2008;46:224232. [PubMed: 17850833]

Sowell ER, Thompson PM, Welcome SE, Henkenius AL, Toga AW, Peterson BS. Cortical abnormalities in children and adolescents with attention-deficit hyperactivity disorder. Lancet 2003;362:1699_ 1707. [PubMed: 14643117]

Suskauer SJ, Simmonds DJ, Fotedar S, Blankner JG, Pekar JJ, Denckla MB, Mostofsky SH. Functional magnetic resonance imaging evidence for abnormalities in response selection in attention deficit hyperactivity disorder: differences in activation associated with response inhibition but not habitual motor response. Journal of Cognitive Neuroscience 2008;20:478-493. [PubMed: 18004945]

Suzuki M, Zhou SY, Takahashi T, Hagino H, Kawasaki Y, Niu L, Matsui M, Seto H, Kurachi M. Differential contributions of prefrontal and temporolimbic pathology to mechanisms of psychosis. Brain 2005;128:2109-2122. [PubMed: 15930048]

Tekin S, Cummings JL. Frontal-subcortical neuronal circuits and clinical neuropsychiatry: an update. Journal of Psychosomatic Research 2002;53:647-654. [PubMed: 12169339]

Wechsler, D. Wechsler Intelligence Scale for Children-III. The Psychological Corporation; San Antonio, TX: 1991.

Wechsler, DL. Wechsler Individual Achievement Test. The Psychological Corporation; San Antonio, TX: 1992.

Wechsler, DL. Wechsler Individual Achievement Test-II. The Psychological Corporation; San Antonio, TX: 2002.

Wechsler, DL. Wechsler Intelligence Scale for Children. Vol. 4. The Psychological Corporation; San Antonio, TX: 2003.

Wible CG, Shenton ME, Fischer IA, Allard JE, Kikinis R, Jolesz FA, Iosifescu DV, McCarley RW. Parcellation of the human prefrontal cortex using MRI. Psychiatry Research 1997;76:29-40. [PubMed: 9498307]

Willcutt EG, Doyle AE, Nigg JT, Faraone SV, Pennington BF. Validity of the executive function theory of attention-deficit/hyperactivity disorder: a meta-analytic review. Biological Psychiatry 2005;57:1336-1346. [PubMed: 15950006]

Wodka EL, Mahone EM, Blankner JG, Larson JC, Fotedar S, Denckla MB, Mostofsky SH. Evidence that response inhibition is a primary deficit in ADHD. Journal of Clinical and Experimental Neuropsychology 2007;29:345-356. [PubMed: 17497558]

Wolosin SM, Richardson ME, Hennessey JG, Denckla MB, Mostofsky SH. Abnormal cerebral cortex structure in children with ADHD. Human Brain Mapping. 2007

Yamasue H, Iwanami A, Hirayasu Y, Yamada H, Abe O, Kuroki N, Fukuda R, Tsujii K, Aoki S, Ohtomo $\mathrm{K}$, Kato N, Kasai K. Localized volume reduction in prefrontal, temporolimbic, and paralimbic regions in schizophrenia: an MRI parcellation study. Psychiatry Research 2004;131:195-207. [PubMed: 15465289]

Zilles K, Schleicher A, Langermann C, Amunts K, Morosan P, Palomero-Galagher N. Quantative analysis of the sluci in the human cerebral cortex: development, regional heterogeneity, gender differences, asymmetry, intersubject variability and cortical architecture. Human Brain Mapping 1997;5:218221. 
Zilles K, zur Nieden K, Schleicher A, Traber J. A new method for quenching correction leads to revisions of data in receptor autoradiography. Histochemistry 1990;94:569-578. [PubMed: 2177745] 


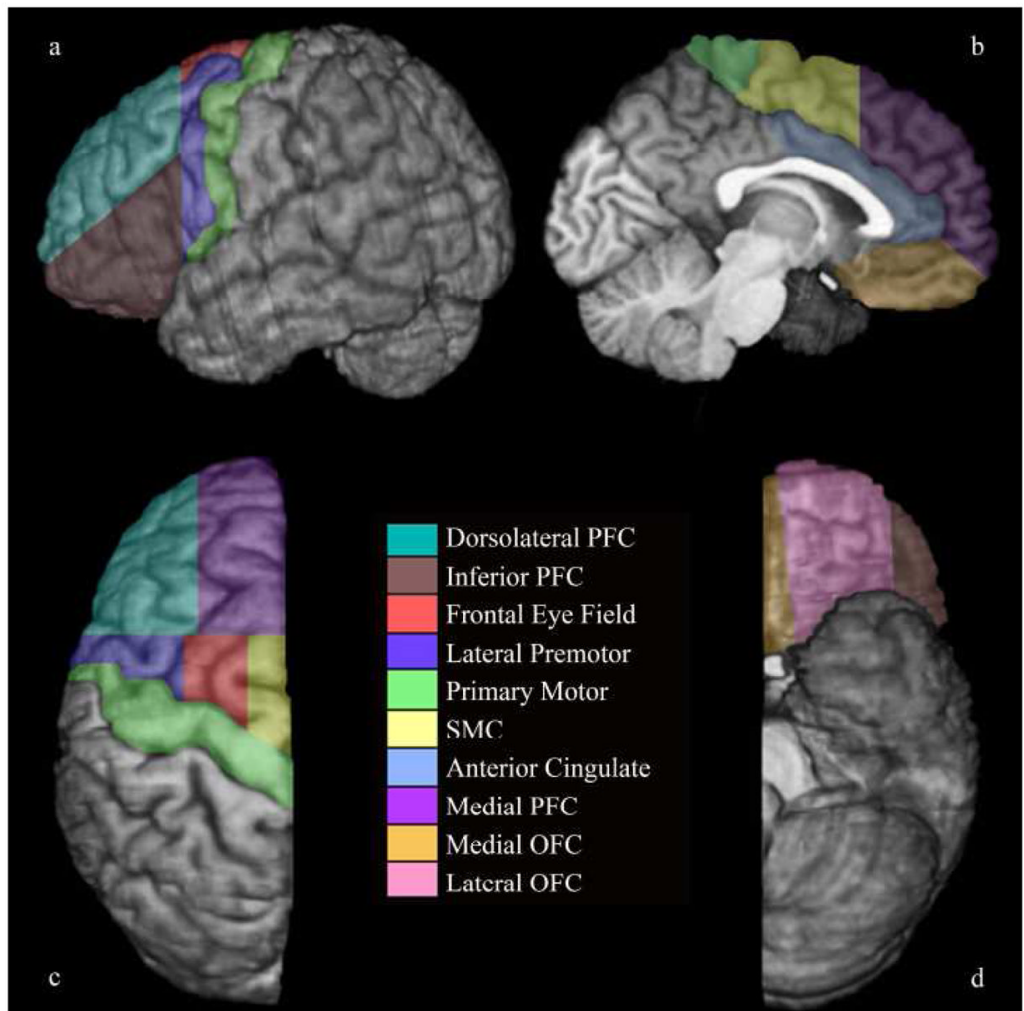

Figure 1.

Parcellated left frontal lobe of an 11.5 year-old boy on a) lateral, b) medial, c) dorsal and d) ventral surfaces. The deep white matter region is not visible on the surface of the brain; a sample DWM can be seen in Figure 2. 


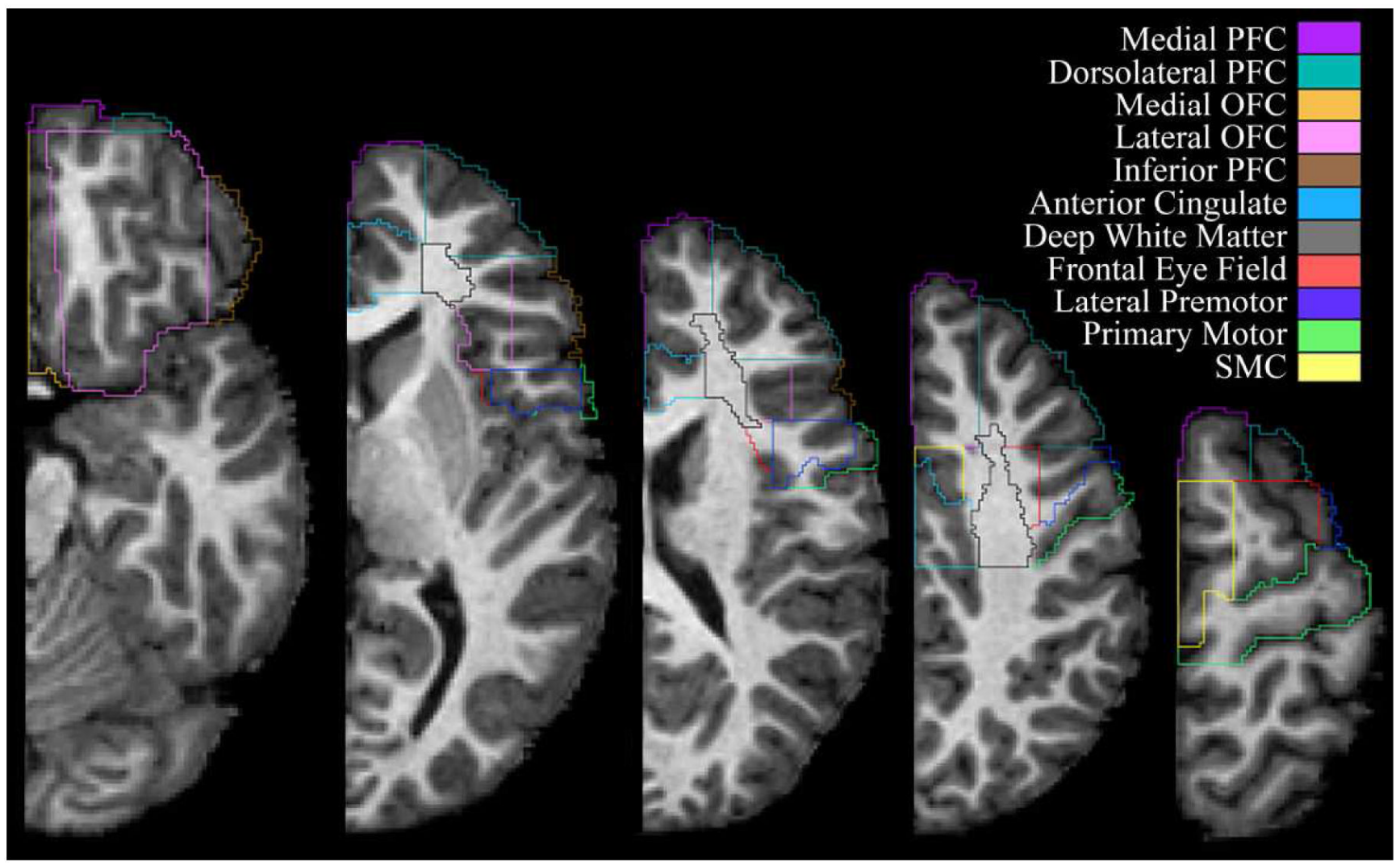

Figure 2.

A sample frontal lobe parcellation in a series of axial cross-sections. Images from left to right move from more inferior to more superior positions in the brain. 

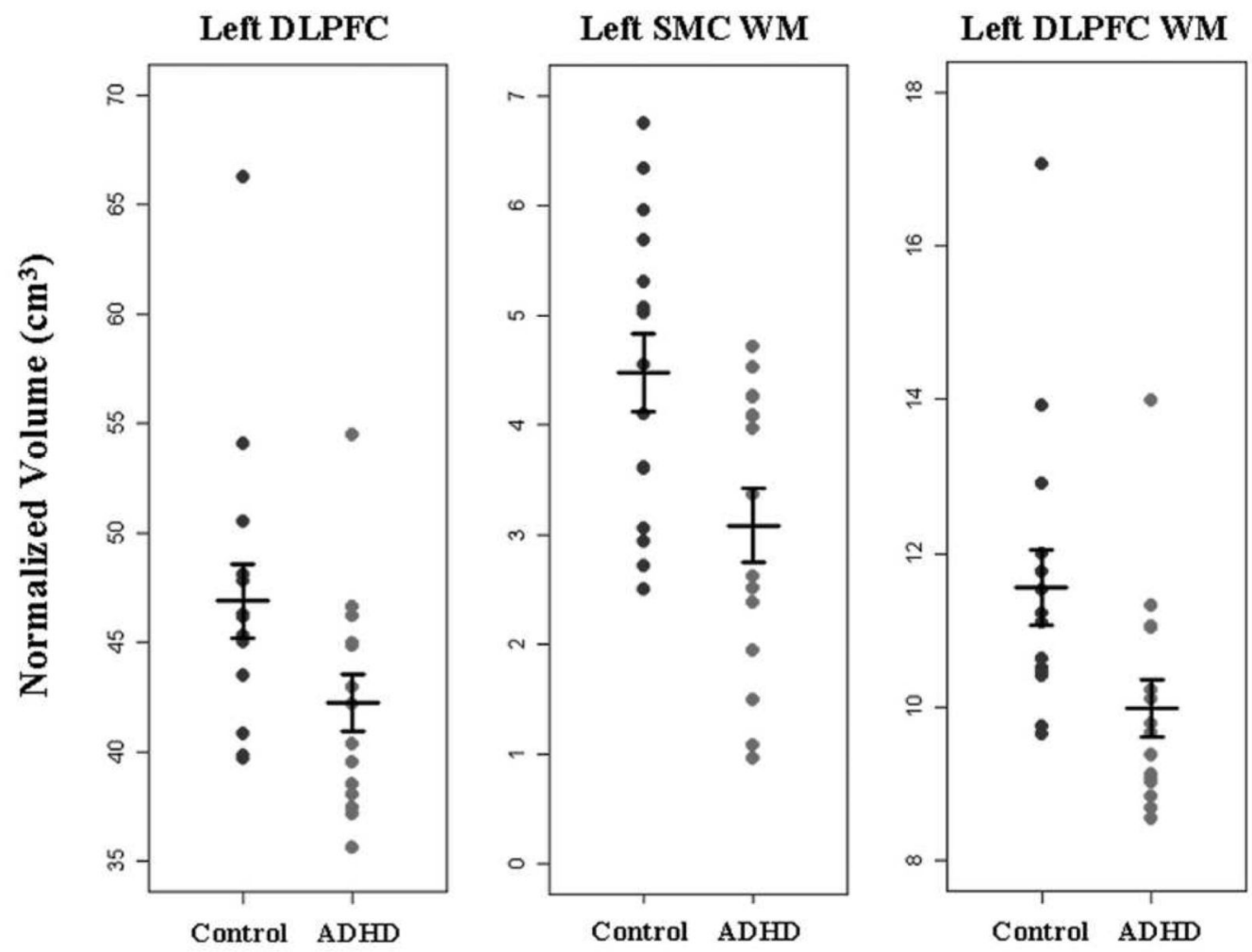

Figure 3.

Plots of volume after normalization for TCV, grouped by diagnosis for left DLPFC, left SMC WM and left DLPFC WM. Horizontal bars indicate the mean and standard error for each group. Findings showed significantly reduced volume in ADHD in all three regions, with the effect of outliers mitigated by the use of non-parametric analyses. 
TABLE 1

Intra- and inter-rater intraclass correlation coefficients for frontal lobe and sub-regions

\begin{tabular}{|c|c|c|}
\hline Region & Intra-rater ICC & Inter-rater ICC \\
\hline Frontal Lobe & 0.998 & 0.997 \\
\hline \multicolumn{3}{|c|}{ Left Hemisphere } \\
\hline L Primary Motor & 0.991 & 0.992 \\
\hline L Ant Cingulate & 0.997 & 0.997 \\
\hline L Deep White & 0.975 & 0.959 \\
\hline L SMC & 0.954 & 0.946 \\
\hline L FEF & 0.980 & 0.802 \\
\hline L LPM & 0.956 & 0.916 \\
\hline L Medial PFC & 0.968 & 0.957 \\
\hline L DLPFC & 0.986 & 0.950 \\
\hline L Inferior PFC & 0.778 & 0.798 \\
\hline L Lateral OFC & 0.934 & 0.961 \\
\hline L Medial OFC & 0.976 & 0.894 \\
\hline \multicolumn{3}{|c|}{ Right Hemisphere } \\
\hline R Primary Motor & 0.930 & 0.978 \\
\hline R Ant Cingulate & 0.981 & 0.979 \\
\hline R Deep White & 0.948 & 0.907 \\
\hline R SMC & 0.965 & 0.895 \\
\hline R FEF & 0.975 & $0.724^{*}$ \\
\hline R LPM & 0.877 & 0.861 \\
\hline R Medial PFC & 0.996 & 0.980 \\
\hline R DLPFC & 0.972 & 0.979 \\
\hline R Inferior PFC & 0.928 & 0.839 \\
\hline R Lateral OFC & 0.992 & 0.987 \\
\hline R Medial OFC & 0.888 & 0.925 \\
\hline
\end{tabular}

* indicates that 4 of 5 subjects were used in determining ICC for the R FEF due to an unusual sulcal configuration in the 5 th subject. 


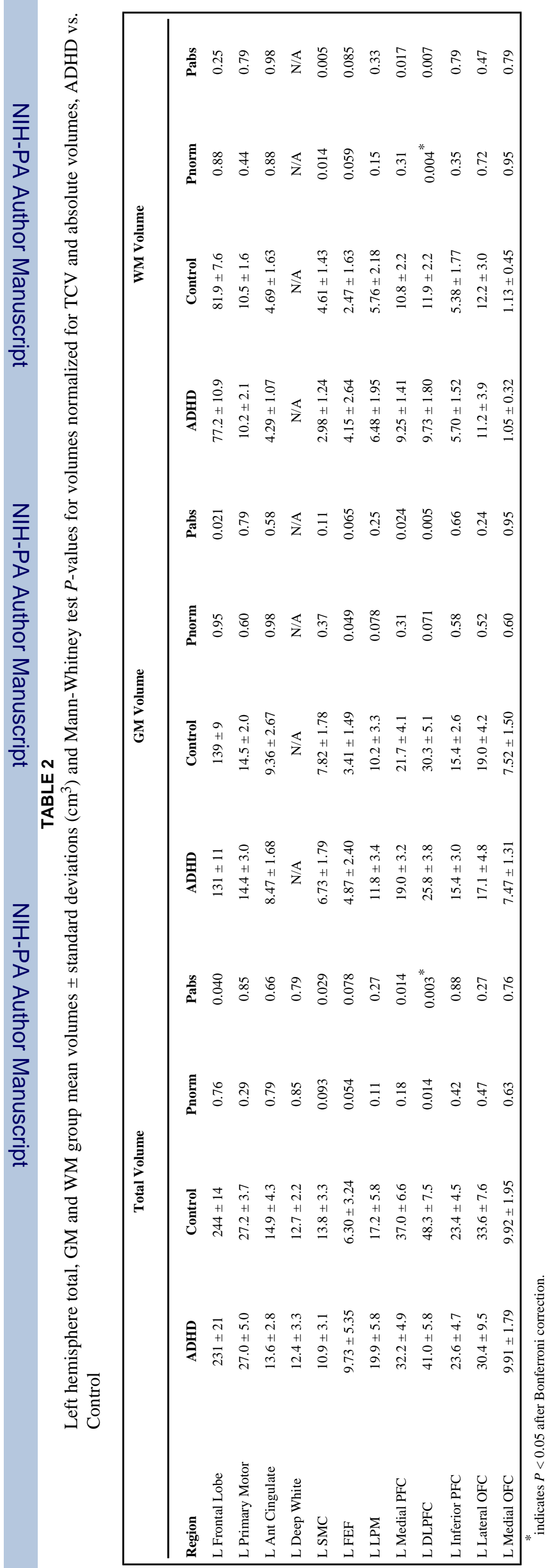




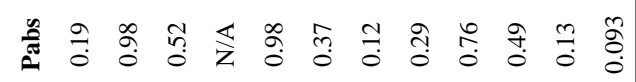

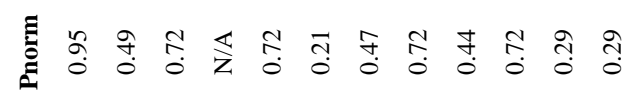
言

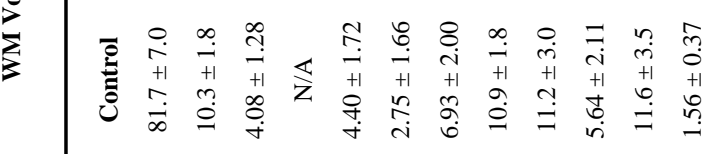

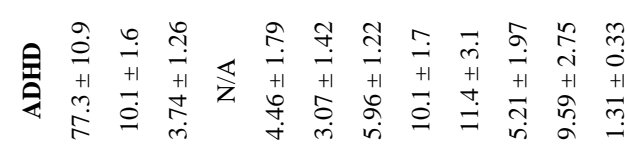

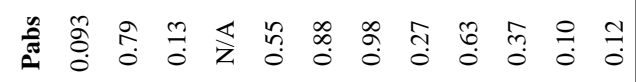

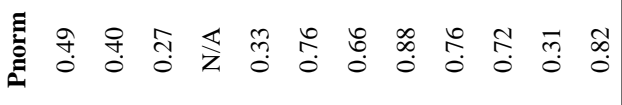

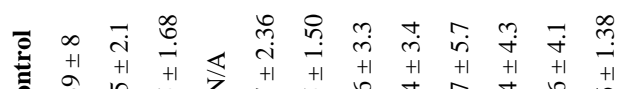

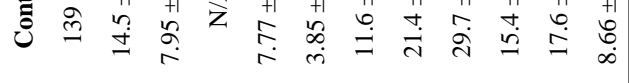

